\title{
An Activity Recognition Algorithm Based on Energy Expenditure Model
}

\author{
Yuhuang Zheng \\ Department of Physics, Guangdong University of Education, Guangzhou, 510303, China \\ zhyhaa@126.com
}

\begin{abstract}
Keywords: Energy Expenditure, Fast Fourier Transform(FFT), Discrete Wavelet Transform (DWT), triaxial accelerometer

Abstract. Human activity recognition via triaxial accelerometers can provide valuable information to evaluate functional abilities. In this paper, we present an accelerometer sensor-based approach for human activity recognition. Our proposed recognition method uses a model of Activity Energy Expenditure to recognize six activities. The classifier utilizes Fast Fourier transform (FFT) and Discrete Wavelet Transform (DWT) algorithms to get energy expenditures of different activities. Every activity is recognized by the max amplitude and its frequency, 1-D decomposition energy of triaxial accelerometer signals. This activity recognition method can recognize six activities with an average accuracy of $90 \%$ using only a single triaxial.
\end{abstract}

\section{Introduction}

Recently, activity recognition is an emerging field of research and is one of the challenges for pervasive computing. A typical application for activity recognition is in the health care. Activity recognition also is an important research issue in building a pervasive and smart environment to provide personalized support.

Computer vision-based techniques and the body-fixed accelerators are the main methodologies designed for activity recognition. Computer vision-based techniques for activity recognition should be conducted in a well-controlled environment and be due to limitations of the environment. However, they may fail notably in an environment with the clutter and variable lighting [1-3]. The body-fixed accelerators offer a practical and relatively low cost method to catch the human motion.

The existing literature demonstrates many studies on activity recognition using accelerometers. Many researchers have used particular devices to collect the raw accelerometer data for a set of movements and have employed a wide variety of activity recognition algorithms. [4-6]

These studies above have considered activity recognition as a supervised learning problem. Training dataset is the main step for the supervised learning. But it is hard to get training dataset. Firstly, it is a tedious and difficult work to generate a training dataset. It is a laborious task for analysis and labels the whole huge sensor records. Secondly, the same activity may have different sensor data for different persons to train motion model. The model can classify one person's activity accurately, but it could not be satisfying results of this model for another. At this time, the training dataset and the activity classifier should be regenerated. For these reasons, we need to find a new approach of human activity recognition without training model processing for new persons. [7]

In this paper, we aim to overcome the limitations of existing physical-activity recognition system and intend to develop a novel method that is capable of recognizing a set of daily physical activities using only a single triaxial accelerometer. This method uses the max amplitude and its frequency, 1-D decomposition energy of triaxial accelerometer signals as features of activities. Different activities have different ranges of feature threshold parameters. If feature data are within a specified threshold range, the activity is recognized. The computation of features data is based on Fast Fourier Transform (FFT) and Discrete Wavelet Transform (DWT) algorithm. This method is capable of recognizing six physical activities of daily living with a high recognition rate 


\section{The Human Activity Energy Expenditure Model}

About dynamic activities, [8] represents that energy expenditure for a person to walk $1 \mathrm{~m}$ on the flat is about $0.17 \mathrm{~kJ}$, to run $1 \mathrm{~m}$ is about $0.25 \mathrm{~kJ}$, to jump $1.2 \mathrm{~m}$ horizontally consumed on average around 3.8 $\mathrm{kJ}$. If one person walks, runs and jumps at the same velocity $50 \mathrm{~m} / \mathrm{min}$, he will cost energy $8.5 \mathrm{~kJ} / \mathrm{min}$, $12.5 \mathrm{~kJ} / \mathrm{min}$ and $158.3 \mathrm{~kJ} / \mathrm{min}$ respectively. Energy expenditure to jump a given distance is about 18.6 times greater than to walk the same distance. Running a given distance requires about 1.5 times the energy expenditure of walking the same distance.

About static activities, [9] and [10] represent energy expenditure of sleeping is $0.38 \mathrm{~kJ} / \mathrm{min}$, sitting is $4.27 \mathrm{~kJ} / \mathrm{min}$ and standing is $5.69 \mathrm{~kJ} / \mathrm{min}$. In the same given time period, energy expenditure of sleeping is the least and standing requires the most energy. Obviously, any static activity costs less energy than on of dynamic activities.

From what has been discussed above, we draw the conclusion that Energy Expenditure (EE) relationship is expressed among the six activities by Eq. 1.

$$
E E_{\text {sleep }}<E E_{\text {sit }}<E E_{\text {stand }}<E E_{\text {walk }}<E E_{\text {run }}<E E_{\text {jump }}
$$

According to the law of conservation of energy, if someone's energy expenditure is converted into his kinetic energy, then we get Eq. 2.

$$
E E=\frac{1}{2} m v^{2}=\frac{1}{2} m\left(v_{0}+a t\right)^{2}
$$

Because the initial velocity $v_{0}$, someone's mass $m$ and his motion time period $t$ is known, we can get the energy expenditure if we know his acceleration data $a$. We can use acceleration data $a$ provided by a 3-axis accelerometer to compute $E E$ and to classify the six activities.

\section{Features Selection of Energy Expenditure}

In our approach, according to Energy Expenditure of walking, running, jumping, standing, sitting and sleeping, we use the Fast Fourier transform to extract the max magnitude and its frequency information as activity features which magnitude and frequency information products by a triaxial acceleration vector. The tri-axial acceleration vector $A(t)$ is Eq. 3 .

(3)

$$
A(t)=x_{a}(t) \vec{e}_{x}+y_{a}(t) \vec{e}_{y}+z_{a}(t) \vec{e}_{z}, t=1, \cdots N
$$

where $x_{a}(t), y_{a}(t)$, and $z_{a}(t)$ represent the $t$ acceleration sample of the $x, y$, and $z$ axis. This feature is independent of the orientation of the sensing device, and measures the instantaneous intensity of human movements at index $t$.

We compute the max magnitude and use them as two features given by $M_{\text {mag_max }}$ and $f_{\text {mag_max }}$, such as Eq. 4 - Eq. 8.

$$
\begin{aligned}
& \operatorname{triA}=\{|A(1)|, \cdots,|A(N)|\} \\
& M_{\text {mag }}(k)=2\left|\sum_{j=1}^{\frac{N}{2}+1} \operatorname{tri} A(j) \omega_{N}^{(j-1)(k-1)}\right|, k=1, \cdots,\left(\frac{N}{2}+1\right) \\
& f_{\text {mag }}(k)=\frac{(k-1)}{N} f_{\text {sample }}, k=1, \cdots,\left(\frac{N}{2}+1\right) \\
& M_{\text {mag_max }}=\max \left[M_{\text {mag }}(k)\right], k \geq 2 \\
& f_{\text {mag_max }}=f_{\text {mag }}\left(k_{\text {max }}\right), \text { where } M_{\text {mag } \_ \text {max }_{1}}=\max \left[M_{\text {mag }}\left(k_{\text {max }}\right)\right]
\end{aligned}
$$

$f_{\text {sample }}$ is the sampling frequency. 
If $M_{\text {mag_max }}$ is a too small value, this means that the signal is produced by static activities. At this time, $M_{\text {mag_max }}$ cannot distinguish the signal among static activities. This signal should be extracted using wavelet decomposition to get the energy for the triaxial accelerometer data. Let the vector $E_{t}$ that contains the energy distribution of each decomposed vector of $A(t)$, and the wavelet energy of the signal triA is Eq. 9.

$$
E_{\text {wavelet }}=\sqrt{\frac{1}{N} \sum_{t=1}^{N} E_{t}^{2}}
$$

\section{Experiment}

The activities recognition dataset is the USC Human Activity. The activity dataset included the six activities and collected data from 14 subjects. In order to capture the day-to-day activity variations, each subject was called upon to perform 5 trials for each activity on different days at various indoor and outdoor locations. Although the duration of each trial varies across different activities, it was long enough to capture all the information of each performed activity[11].

The test data include all trials in USC dataset and four episodes of each trial are selected randomly. Every episode has a duration of 5 seconds. It means that the opportunity of the algorithm performance test for one activity is no less than 280 times. The test statistic are calculated from the 2800 experimental episodes and the results are shown in Table 1.

Table 1. accuracy rate of the activity recognition algorithm

\begin{tabular}{|c|c|c|c|c|c|c|c|c|c|c|}
\hline \multirow[b]{2}{*}{$\begin{array}{l}\text { input } \\
\text { output }\end{array}$} & \multicolumn{5}{|c|}{ Walking } & \multirow[b]{2}{*}{ Running } & \multirow[b]{2}{*}{ Jumping } & \multirow[b]{2}{*}{ Standing } & \multirow[b]{2}{*}{ Sitting } & \multirow[b]{2}{*}{ Sleeping } \\
\hline & $\begin{array}{c}\text { Forwar } \\
\text { d }\end{array}$ & Left & Right & Up & Down & & & & & \\
\hline Walking & $97.1 \%$ & $88.6 \%$ & $100.0 \%$ & $80.0 \%$ & $77.1 \%$ & $0.0 \%$ & $7.1 \%$ & $0.0 \%$ & $0.0 \%$ & $0.0 \%$ \\
\hline Running & $0.0 \%$ & $0.0 \%$ & $0.0 \%$ & $4.3 \%$ & $2.9 \%$ & $92.9 \%$ & $2.9 \%$ & $0.0 \%$ & $0.0 \%$ & $0.0 \%$ \\
\hline Jumping & $1.4 \%$ & $11.4 \%$ & $0.0 \%$ & $8.6 \%$ & $12.9 \%$ & $7.1 \%$ & $90.0 \%$ & $0.0 \%$ & $0.0 \%$ & $0.0 \%$ \\
\hline Standing & $0.0 \%$ & $0.0 \%$ & $0.0 \%$ & $7.1 \%$ & $7.1 \%$ & $0.0 \%$ & $0.0 \%$ & $80.0 \%$ & $1.4 \%$ & $0.0 \%$ \\
\hline Sitting & $1.4 \%$ & $0.0 \%$ & $0.0 \%$ & $0.0 \%$ & $0.0 \%$ & $0.0 \%$ & $0.0 \%$ & $17.1 \%$ & $98.6 \%$ & $10.0 \%$ \\
\hline Sleeping & $0.0 \%$ & $0.0 \%$ & $0.0 \%$ & $0.0 \%$ & $0.0 \%$ & $0.0 \%$ & $0.0 \%$ & $2.9 \%$ & $0.0 \%$ & $90.0 \%$ \\
\hline \multirow{2}{*}{ accuracy } & $97.1 \%$ & $88.6 \%$ & $100.0 \%$ & $80.0 \%$ & $77.1 \%$ & \multirow{2}{*}{$92.9 \%$} & \multirow{2}{*}{$90.0 \%$} & \multirow{2}{*}{$80.0 \%$} & \multirow{2}{*}{$98.6 \%$} & \multirow{2}{*}{$90.0 \%$} \\
\hline & & & $88.6 \%$ & & & & & & & \\
\hline
\end{tabular}

Table 1 reports the results of six activities recognition accuracy rates. The average accuracy rate is $90 \%$ approximately and it is acceptable for a person-independent activity recognition algorithm. When one person walks up or down, the activity recognition algorithm has a 20 percent chance to recognize running, jumping or standing. If someone goes up/downstairs too fast, the algorithm has a tendency to identify the activity as running or jumping. If someone goes up/downstairs slowly, the activity would be recognized as standing. If a person just stands and does nothing, the activity would have a good chance of identifying the activity as sitting or sleeping. Abnormal activity will affect the accuracy rate of the activity recognition algorithm. We will attempt to get higher recognition accuracy by using more features in future research.

\section{Conclusion}

The aim of this paper is to provide an accurate and robust human activity recognition algorithm. The algorithm uses FFT and wavelet decomposition extract features of six typical activities from triaxial 
acceleration data. We build the model of Activity Energy Expenditure based on human physiology. The max magnitude and its corresponding frequency, wavelet decomposition energy of triaxial acceleration data are used as indexes of energy expenditure. The activity recognition algorithm is effective in recognizing six daily physical activities with an average accuracy of $90 \%$. It is able to distinguish walking, running, jumping, standing, sitting and sleeping activities using only a single tri-axial accelerometer.

The next stage of our research will consist of two parts. Firstly, algorithms are improved to recognize these activities and the user will not have to worry that sensors are placed in the right position for the activities to be detected correctly. Those mean human activity recognition can be performed in a natural way. Secondly, more typical activities should be recognized and new features of different activities are considered. We will optimize the activity recognition algorithm by different benchmark datasets.

\section{Acknowledgements}

This work was supported in part by Appropriative Researching Fund for Professors and Doctors, Guangdong University of Education under Grant 11ARF04 and Guangdong Provincial Department of Education under Grant 2013LYM_0063 and 2014GXJK161.

\section{References}

[1] J.K. Aggarwal, L. Xia: Pat. Rec. Letters. Vol.48 (2014), p.70

[2] J. Hernández, R. Cabido, A. S. Montemayor, J.J. Pantrigo: Exp. Sys. Vol.31 (2014), p.345

[3] J. Yin, G. Tian, Z. Feng, J. Li: Comput. and Elec. Eng. Vol.40 (2014), p.538

[4] O. D. Incel, M. Kose, C. Ersoy: Bio. Nano. Sci. Vol.3 (2013), p.145

[5] Y. Liang, X. Zhou, Z. Yu, B. Guo: Mobile Netw. Appl. Vol.19 (2014), p.303

[6] J. P. Varkey, D. Pompili, T. A. Walls: Pers. Ubiquit. Comput. Vol. 16 (2012), p.897

[7] Y. Kwon, K. Kang, C. Bae: Exp. Sys. with Appl. Vol. 41 (2014), p.6067

[8] S. R. L. Coward, L. G. Halsey: Bio. Open. Vol. 3 (2014), p.815

[9] C.Reiff, K.Marlatt, D. R. Dengel: J. of Phys. Act. and Heal. Vol. 9 (2012), p.1009

[10]C.M. Jung, E.L. Melanson, E.J. Frydendall1, L.Perreault, R.H. Eckel, K.P.Wrigh: J. Physiol. Vol. 589 (2011), p.235

[11]M. Zhang, A.A. Sawchuk, USC-HAD: A Daily Activity Dataset for Ubiquitous Activity Recognition Using Wearable Sensors, ACM Inter. Con. on Ubiquit. Comput. (UbiComp) Work. on Situation, Activity and Goal Awareness (SAGAware), Pittsburgh, Pennsylvania, USA, Sept. 2012. 